\title{
Microscopic optoelectronic defectoscopy of solar cells
}

\author{
P. Škarvada, P. Tománek, P. Koktavý and D. Dallaeva \\ Brno University of Technology, Faculty of Electrical Engineering and Communication, Physics Department, \\ Technická 8, 61600 Brno, Czech Republic
}

\begin{abstract}
Scanning probe microscopes are powerful tool for micro- or nanoscale diagnostics of defects in crystalline silicon solar cells. Solar cell is a large p-n junction semiconductor device. Its quality is strongly damaged by the presence of defects. If the cell works under low reverse-biased voltage, defects emit a light in visible range. The suggested method combines three different measurements: electric noise measurement, local topography and near-field optical beam induced current and thus provides more complex information. To prove its feasibility, we have selected one defect (truncated pyramid) in the sample, which emitted light under low reverse-biased voltage.
\end{abstract}

\section{Introduction}

Solar energy, radiant light and heat from the sun, has been harnessed by humans since ancient times using a range of ever-evolving technologies. Solar energy technologies include solar heating, solar photovoltaics, solar thermal electricity and solar architecture, which can make considerable contributions to solving some of the most urgent problems the world now faces.

Solar technologies are broadly characterized as either passive solar or active solar depending on the way they capture, convert and distribute solar energy. Active solar techniques include the use of photovoltaic panels and solar thermal collectors to harness the energy. Passive solar techniques include orienting a building to the Sun, selecting materials with favorable thermal mass or light dispersing properties, and designing spaces that naturally circulate air.

The crystalline silicon is still most widespread devices used in solar cells. Solar cells are large $p-n$ junction semiconductor devices. However, their quality and efficiency is strongly affected adversely by a variety of defects or imperfections of diverse nature. Some of them are troublesome for a good function of solar plant installation; others could be neglected [1].

Generally, widely used electrical measurements represent basic characterization methods of silicon solar cells [2]. Due to their integral nature which requires the measurement over whole cell or panel, they only inform us about the presence of defects in the structure.

With the development of charge coupled device detectors, a lock-in thermography, a considerable number of fast imaging methods and applications in solar cell characterization has been developed $[3,4]$. These methods are mainly used in in-situ characterization and process control for mass wafer production.

Nevertheless, there are still many opened questions in field of local light emission, material defects and their relations. Local defects in the $p-n$ junction may be associated with structural imperfections (such as grain boundaries, dislocations, scratches), chemical impurities, higher concentrations of donors and acceptors $[5,6]$.

In this paper we present results of local detection, localization and comparison of several kinds of defects in monocrystalline silicon using different mapping and nonmapping electrical and optical techniques. To prove the feasibility of these methods, we have chosen among various examples a site with truncated etched silicon pyramid, which emitted light under low reverse-biased voltage.

\section{Defectoscopy of solar cells}

Monocrystalline silicon, due to its structure, is very interesting material for local investigation and comparison of used methods as SEM visualization of defects, electric noise measurement and local topography and near-field optical beam induced current measurements [7-10].

There are two classes of defects in silicon:

- material defects provided by imperfections or irregularity in crystal structure (point, line, square or volume defects),

- processing defects due to the imperfection of materials during processing.

Presently, following measuring methods are used for investigation on prefabricated Si wafers:

- Solar simulator with tester

- Noise spectroscopy

- Light Beam Induced Current (LBIC)

- Electroluminescence

- Photoluminescence

- Lock-in thermography (LIT).

First the electrical noise measurement on Si sample allows detect a presence of low-voltage breakdowns in defect sites of $p-n$ junctions (figure 1). This noise current 
is a first symptom which refers to the presence of defects in the Si wafer [6].

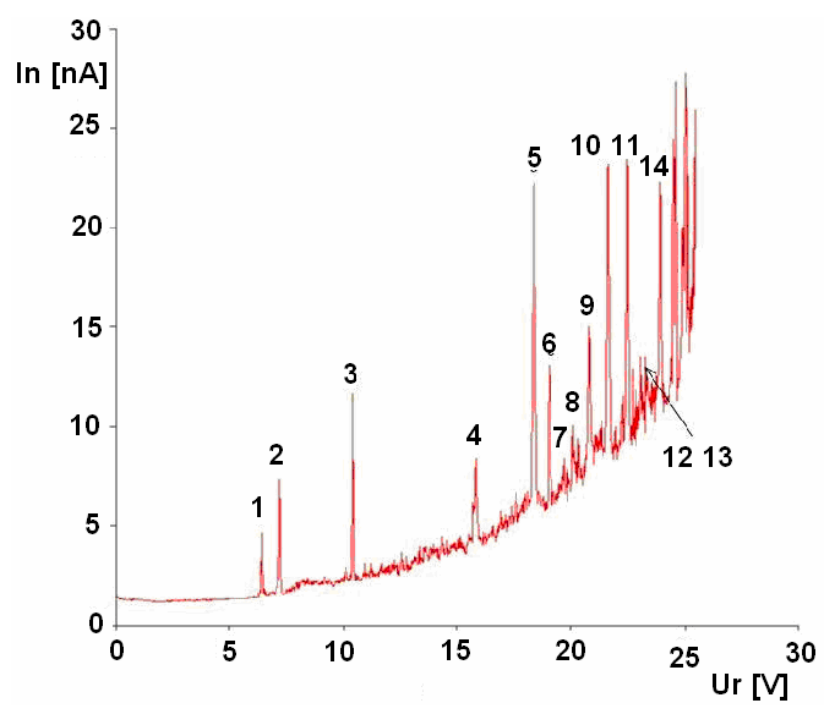

Fig. 1. Noise current vs reverse-bias voltage of solar cell with defects [7]

Forward- and reverse-biased voltages on p-n junction can be used here. However, both methods provide different results. While first one needs higher voltage, and it informs us about overall cell structure, low reversebiased voltage allows localize a position of the defect. If this position is roughly known, one can then proceed to local investigation of its electrical, optical and thermal characteristics. A combination of reverse-biased light emission a LBIC (local beam induced current) method goes towards more precise defect localization in the cell (figure 2).

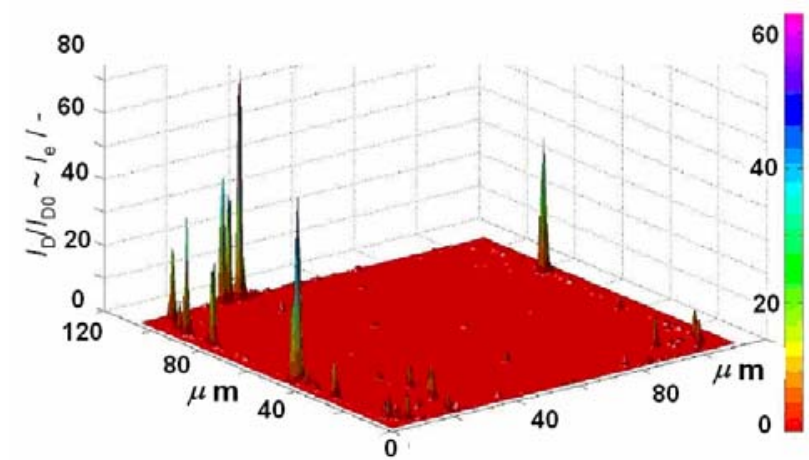

Fig. 2. Localization of defects in solar cell wafer using reversebias light emission in combination with LBIC

$$
(U r=5.9 \mathrm{~V})
$$

\section{Experimental setup}

The experimental setup consists of modified Ntegra Solaris Scanning near-field optical microscope (SNOM) (with aperture or apertureless) (NT-MDT, Zelenograd, Russia) (figure 3) allowing simultaneous measurements of topography, reflectivity of solar cells and locally induced photocurrent with a lateral resolution of about $250 \mathrm{~nm}$ (figures 4 and 5).

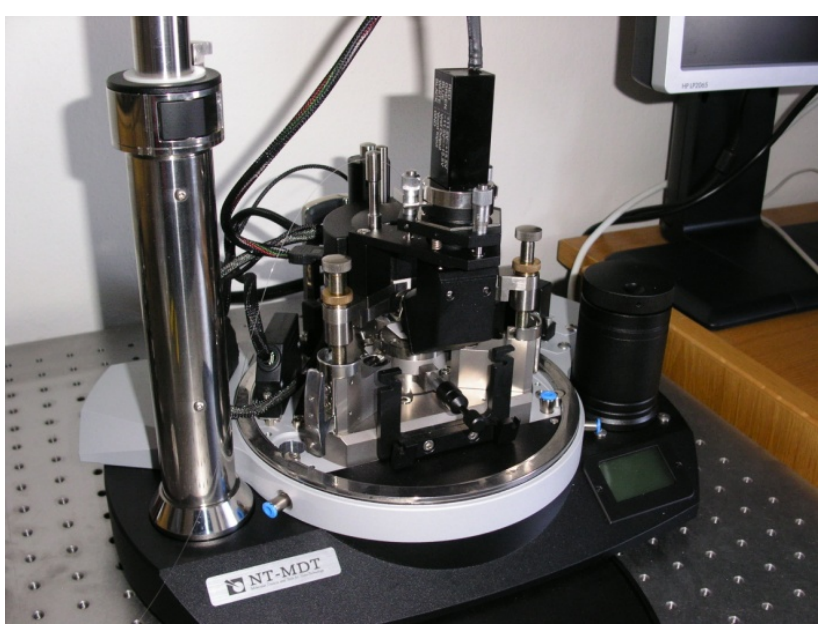

Fig.3. SNOM microscope

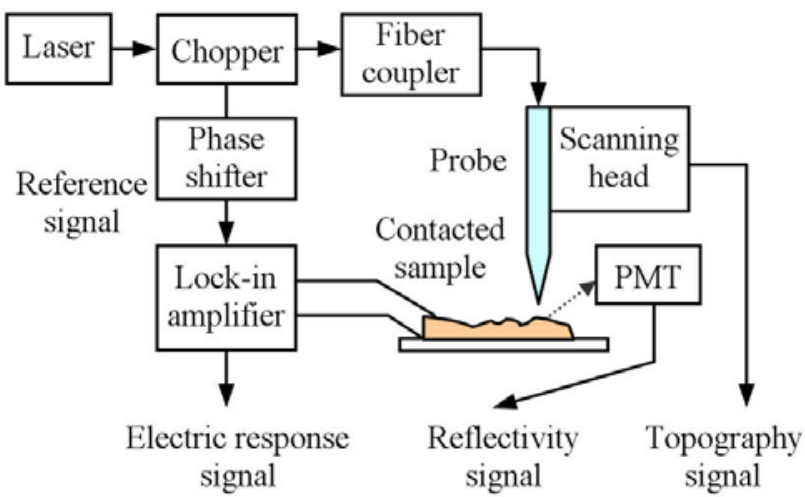

Fig. 4. Experimental setup

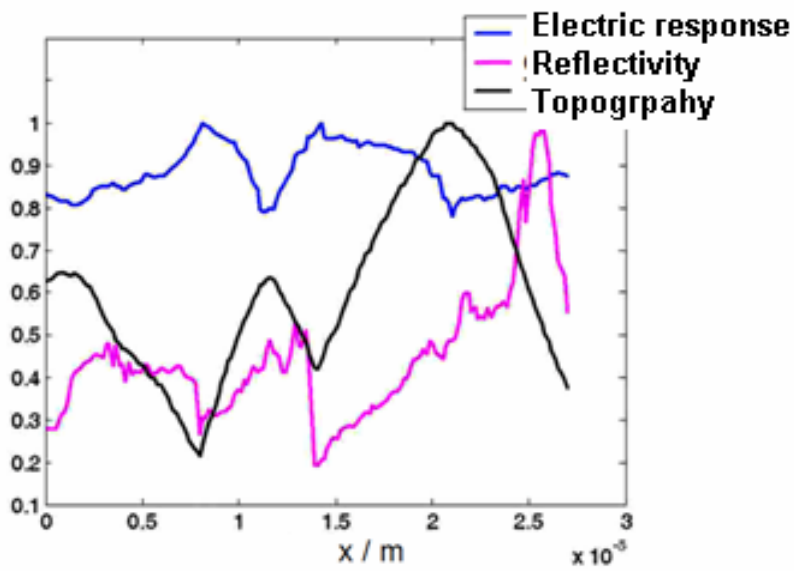

Fig. 5. One line scan over same area of the cell

\section{Results and discussion}

For low reverse bias voltages (here bellow $\mathrm{Ur}=9.0 \mathrm{~V}$ ) the LBIC measurements show that defects do not emit light. Above this threshold, light emission intensity is skyrocketed first with gradual increasing after (figure 6). 


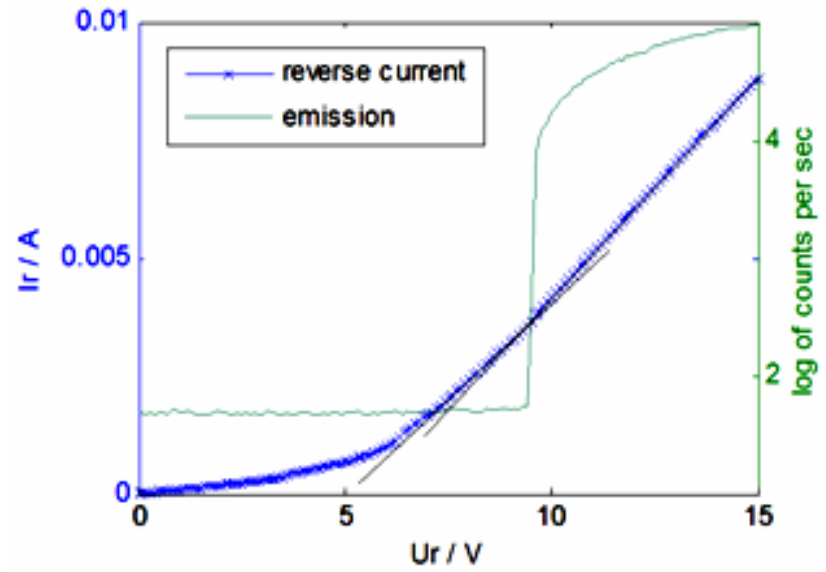

Fig. 6. Local light emission from the defect and reverse part of I-U characteristics $(T=305 \mathrm{~K})$

For relatively high threshold of reverse bias $\left(U_{\mathrm{r}}>5.0 \mathrm{~V}\right)$ the avalanche process is very probable. Approximately in one third of investigated samples it was possible observe together with electrical quantities also a microplasma noise.

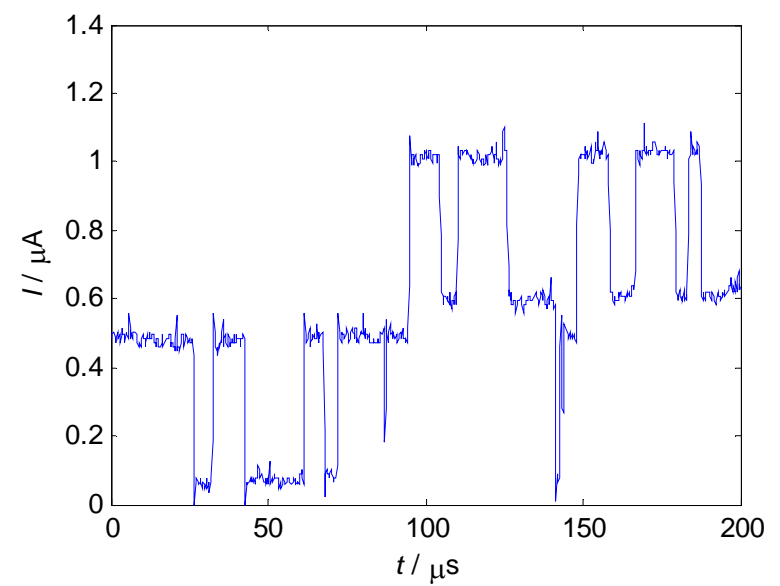

Fig. 7. Bistable character of locally induced photocurrent from the damaged site

The locally induced photocurrent from defected site has a bistable character (figure 7). It is due to the positive thermal characteristics of avalanche breakdown voltage.

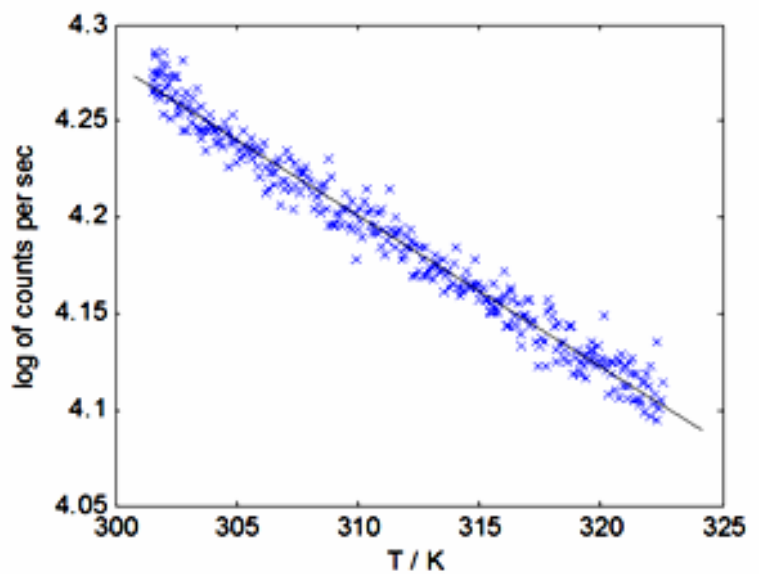

Fig. 8. Thermal dependence of local light emission intensity

$$
\mathrm{U}_{\mathrm{r}}=10.0 \mathrm{~V}
$$

The local avalanche breakdown gives rise to the higher current density and to local temperature of material. Consequently, higher breakdown and a channel occlusion occur. While a channel is closed, the temperature comes down and new local breakdown appears. In the case when the reverse bias is held higher than is characteristic for the material, the ionization of channel is permanent and a bistable noise completely vanished.

There is negative thermal dependence of the light emission there (figure 8).

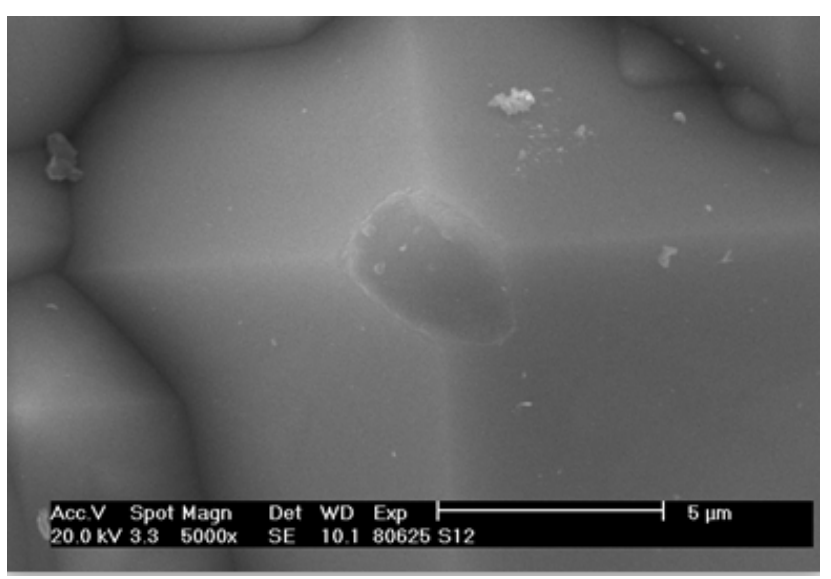

Fig. 9. SEM image of defect on truncated pyramid - p-n junction is destroyed

For a localization of defect sites, a Scanning electron microscope (SEM) could also be used. Here a defect on truncated pyramid with damaged $p-n$ junction was discovered (figure 9). This defect is a source of microplasma (see figure 7).

To confirm this result a corresponding Near-field optically induced current (NOBIC) results is then shown in figure 10. Very small area of solar cell surface (approx. $150 \mathrm{~nm}$ in diameter) has been excited by laser diode light $(\lambda=532 \mathrm{~nm})$ transmitted through a nanometer-sized $(\mathrm{d}=$ $70 \mathrm{~nm}$ ) aperture in the Ag-coated sharpened single mode fiber probe.

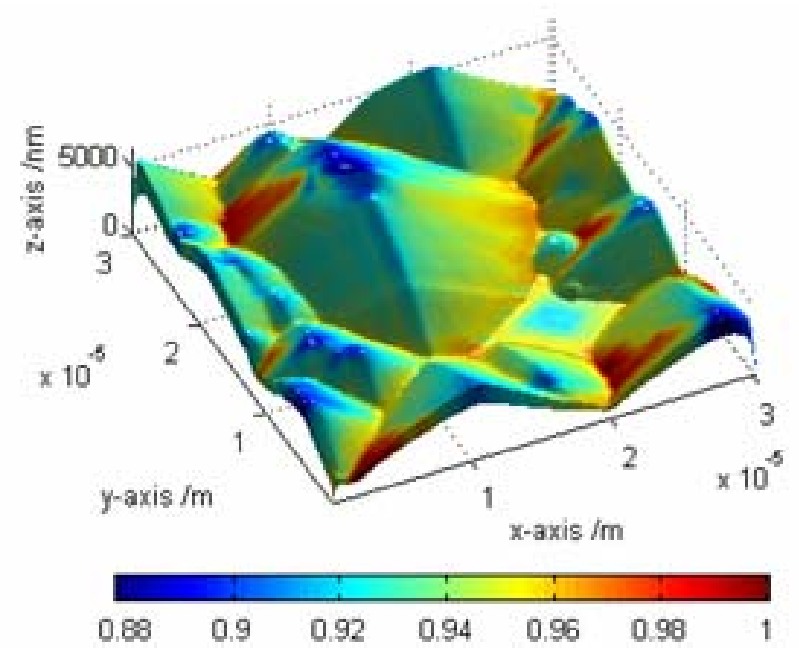

Fig. 10. Relative NOBIC map - a combination of topography and local emission intensity 
Due to the mathematical processing of signal, one can remove the information about topography of sample, and only microplasma noise from the defected site is visualized for $\mathrm{U}_{\mathrm{r}}=9.8 \mathrm{~V}$ (figure 11) and $\mathrm{U}_{\mathrm{r}}=11.0 \mathrm{~V}$ (figure 12).

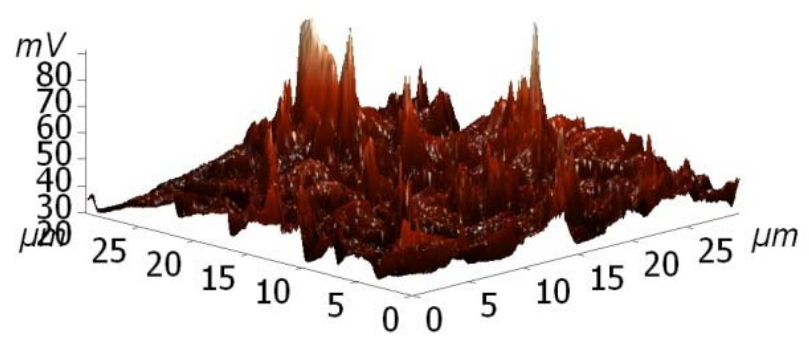

Fig. 11. NOBIC figure shows only microplasma noise light intensity just at the threshold value for $\mathrm{U}_{\mathrm{r}}=9.8 \mathrm{~V}$ when a topographic signal is removed

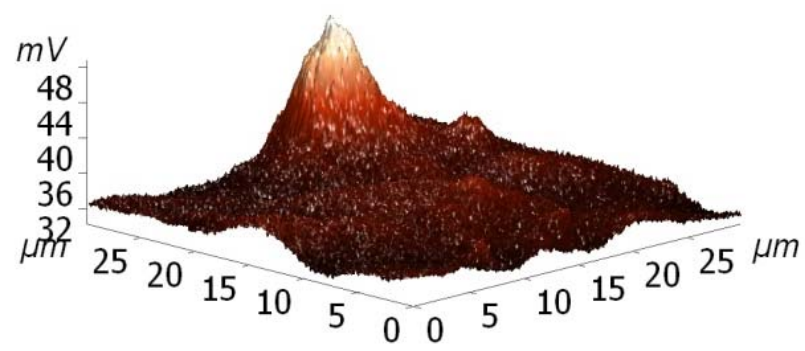

Fig. 12. NOBIC picture of the same area as in figure 11 for $\mathrm{U}_{\mathrm{r}}=11.0 \mathrm{~V}-\mathrm{a}$ topographic signal is removed

\section{Conclusions}

Novel method of microscale localization of solar cell defects based on a combination of local electrical and optical measurement is presented. A combined set-up for apertureless SNOM topography, NOBIC measurement and reflectivity of solar cells with a lateral resolution of about $250 \mathrm{~nm}$ has been established.

The similarity of partial breakdown values and light emission threshold show that the defect has direct connection with local breakdown. It was found that one macro-spot can consist of several micro-spots with diameters bellow $d=3 \mu \mathrm{m}$.

Accuracy of this method depends on actual size of the light spot and on the scanning step of the piezodrive. The accuracy of the combined reflection measurements after calibration is better than $5 \%$.

The method, after little modification can also be applied to other solar cell structures.

\section{Acknowledgments}

This research work has been supported by grant CEITECCentral European Institute of Technology project (CZ.1.05/1.1.00/02.0068) from European Regional Development Fund B, grant GAČR GA P102/10/2013 "Fluctuation processes in $p-n$ junction of solar cells", and by grant of Czech Ministry of Education, Youth and Sports LH11060 "Study of local electric and optical characteristics of solar cells".

\section{References}

1 D.C.Coffey, O.G. Reid, D.B. Rodovsky, G.P. Bartholomew, D.S. Ginger, Nano Lett., 7, 738 (2007)

2 L. Zhang, H. Shen, Zh. Yang, J.Jin, Prog. Photovolt: Res. Appl. 18, 54 (2010)

3 O. Breitenstein, J. Bauer, M. Kittler, T. Arguirov, W. Seifert, Scanning, 30, 331 (2008)

4 M. J. Romero, K. Alberi, I. T. Martin, K. M. Jones, D. L. Young, Y. Yan, C. Teplin, M. M. Al-Jassim, P. Stradins, H. M. Branz, Appl. Phys. Lett. 97, 092107 (2010)

5 S. Rein, in Silicon for Photovoltaic Applications, 85, (Springer Berlin, 2005)

6 D.K. Schroder, Semiconductor Material and Device Characterization (Wiley-IEEE Press, 3rd edition, 2006)

7 P. Koktavy, M. Raska, P. Sadovsky, O. Krcal, AIP Conf. Proc., 922, 141 (2007)

8 P. Tománek, P. Škarvada, R. Macků, L. Grmela, Adv. Opt. Technol., 2010, 805325 (2010)

9 L. Grmela, P. Škarvada, R.Macků, P. Tománek, S. Smith, Sol. Energ. Mat. Sol.C., 96, 118 (2012)

10 P. Škarvada, P. Tománek, L. Grmela, S. Smith Sol. Energ. Mater. Sol. C., 94, 2358 (2010) 